\title{
Interaction effect on topological classification of superconductors in two dimensions
}

\author{
Hong $\mathrm{Yao}^{1,2}$ and Shinsei Ryu ${ }^{3}$ \\ ${ }^{1}$ Institute for Advanced Study, Tsinghua University, Beijing, 100084, China \\ ${ }^{2}$ Department of Physics, Stanford University, Stanford, California 94305, USA \\ ${ }^{3}$ Department of Physics, University of Illinois at Urbana-Champaign, 1110 West Green St, Urbana, IL 61801, USA
}

(Dated: November 12, 2018)

\begin{abstract}
We introduce a new class of superconductors (SCs) in two spatial dimensions with time reversal symmetry and reflection (i.e., mirror) symmetry. In the absence of interactions, topological classes of these SCs are distinguished by an integer-valued $(\mathbb{Z})$ topological invariant. When interactions are included, we show that the topological classification is modified to $\mathbb{Z}_{8}$. This clearly demonstrates that interactions can have qualitative effect on topological classifications of gapped states of matter in more than one dimension.
\end{abstract}

Introduction: Topological phases are fully quantum mechanical states of matter which are not characterized by classical symmetry breaking [1]. While gapped in the bulk, quite often, they are accompanied by gapless excitations at their boundary, signaling highly entangled nature of their ground state. Since the discovery of the integer quantum Hall effect (IQHE) [2], the list of topological phases in nature has been expanded, in particular, by the recent discovery of topological insulators in two and three dimensions (2D and 3D) in systems with strong spin-orbit coupling [3-12], and the identification of ${ }^{3} \mathrm{He}$ $\mathrm{B}$ as a topological SC (superfluid) 13]. Unlike the IQHE, the topological character of these topological insulators and SCs (i.e., the stable gapless edge or surface modes) is protected by time-reversal symmetry (TRS). The presence or absence of a topological distinction among gapped phases for a given set of symmetries and for given spatial dimensions can be studied systematically, and is summarized in the "periodic table" of topological insulators and SCs for non-interacting [14] fermions [15 17].

Since interactions are ubiquitous in real materials, a natural question is whether and how interactions could modify topological classifications obtained for noninteracting systems [18]. In other words, are there cases where interactions qualitatively affect topological classifications? Even though this question has not been fully or thoroughly answered, an exciting progress has been made in Ref. [19], in which Fidkowski and Kitaev explicitly showed that the putative $\mathbb{Z}$ classification of onedimensional (1D) "non-interacting" SCs with unusual time reversal symmetry ("BDI class") is modified to $\mathbb{Z}_{8}$ when interactions are included. This $\mathbb{Z}_{8}$ classification of the 1D BDI SCs with interactions is further illustrated from the study of entanglement spectrum [20, 21]. In more than one dimension, stability of topological classifications in a number of classes of non-interacting topological insulators and SCs is argued from the perspective of low-energy topological response theory [12, 22, 23]. For bosonic (or spin) systems, a systematic approach of constructing symmetry protected topological phases in general spatial dimensions was recently proposed 24]. However, how interactions in fermionic systems in $2 \mathrm{D}$ and 3D can dramatically modify their topological classification obtained from non-interacting fermions remains largely unexplored [25].

In this paper, we partly fill this gap by considering 2D SCs with TRS $\left(\mathcal{T}^{2}=-1\right)$ and reflection symmetry (RS) $\left(\mathcal{R}^{2}=-1\right)$. Note that $\mathcal{T}$ is anti-unitary while $\mathcal{R}$ is unitary. We call SCs with these symmetry properties "DIII+R" SCs. First, we show that the topological classes of these DIII+R SCs are classified by an integervalued $(\mathbb{Z})$ topological invariant at the quadratic level. Such topologically non-trivial SCs are characterized by helical Majorana modes on the system's edge. The number of helical Majorana edge states is protected by the symmetries at the non-interacting level.

We then ask whether those helical gapless Majorana edge modes are stable against interactions while preserving those relevant symmetries in question. As we show later in detail, the helical Majorana edge states in the case of $\mathbb{Z}=8$ topological invariant are unstable against (even weak) interactions - the gapless helical Majorana edge states become gapped while no symmetry is broken in the bulk or the edges of the system. It is worth to stress that all the relevant symmetries are fully preserved while the gap opens in the edge. In other words, the edge of the putative $\mathbb{Z}=8$ system is qualitatively the same as $\mathbb{Z}=0$ systems when symmetry-preserving interactions are included. Because the system's edge we consider respects the same set of symmetries as its bulk, robust edge states could fully encode topological properties of the bulk due to the bulk-edge correspondence. Consequently, the putative $\mathbb{Z}$ topological classification of non-interacting "DIII+R" superconductors in $2 \mathrm{D}$ is reduced $\mathbb{Z}_{8}$ when interactions are considered. A similar model, but with different set of symmetries, was studied independently in Refs. [26, 27].

DIII+R SCs: Two-dimensional SCs with TRS $\mathcal{T}^{2}=$ -1 (symmetry class DIII) have a $\mathbb{Z}_{2}$ topological invariant as shown in the "periodic table". To see this explicitly, it is helpful to study their edge theory. For a $\mathbb{Z}_{2}$ non-trivial DIII SCs in 2D, its generic edge theory is the $1 \mathrm{D}$ helical 
Majorana fermions:

$$
H_{\text {free }}=\int d x\left[\psi_{\uparrow} i \partial_{x} \psi_{\uparrow}-\psi_{\downarrow} i \partial_{x} \psi_{\downarrow}\right],
$$

where $\psi_{\sigma}$ are left/right-moving edge Majorana fermion operators and $\sigma=\uparrow, \downarrow$ are spin index with the following properties under time reversal transformation: $\mathcal{T}^{-1} \psi_{\uparrow} \mathcal{T}=\psi_{\downarrow}$ and $\mathcal{T}^{-1} \psi_{\downarrow} \mathcal{T}=-\psi_{\uparrow}$, which satisfies $\mathcal{T}^{2}=$ -1 . It is clear that the helical edge states above respect the TRS. Moreover, this TRS protects this putative gapless helical edge state since there is only one mass term $i m \psi_{\uparrow} \psi_{\downarrow}$ which breaks the TRS in the edge. However, when there are two such helical gapless modes labeled by $a=1,2$, the putative gapless modes can be fully gapped by time reversal invariant terms $\operatorname{im}\left(\psi_{\uparrow}^{1} \psi_{\downarrow}^{2}+\psi_{\downarrow}^{1} \psi_{\uparrow}^{2}\right)$. Consequently, the topological classification is $\mathbb{Z}_{2}$.

To possibly have a $\mathbb{Z}$ topological classification of DIII $\mathrm{SCs}$ in $2 \mathrm{D}$, further discrete symmetry is needed. Since the helical edge states preserve not only TRS but also the RS $\mathcal{R}$ defined as $\mathcal{R}^{-1} \psi_{\uparrow}(x) \mathcal{R}=\psi_{\downarrow}(-x)$ and $\mathcal{R}^{-1} \psi_{\downarrow}(x) \mathcal{R}=$ $-\psi_{\uparrow}(-x)$ [28], we consider 2D DIII SCs with RS, dubbed as the DIII+R class. Note that $\mathcal{R}^{2}=-1$ as required for spin half-integer fermions. Now, we show that the $\mathrm{DIII}+\mathrm{R}$ SCs in 2D are distinguished by an integer-valued $(\mathbb{Z})$ topological invariant. To see this, we consider $N$ copies (or flavors) of the helical gapless Majorana edge states given by $\sum_{a=1}^{N} \int d x v_{a}\left[i \psi_{\uparrow}^{a} \partial_{x} \psi_{\uparrow}^{a}-i \psi_{\downarrow}^{a} \partial_{x} \psi_{\downarrow}^{a}\right]$, where $a=1, \ldots, N$ are the "flavor" indices (which is preserved by both the time-reversal or reflection transformations) and $v_{a}$ are the Fermi velocities. We then write down the most general mass term

$$
i \psi_{\uparrow}^{a} M_{a b} \psi_{\downarrow}^{b}, \quad M_{a b}^{*}=M_{a b},
$$

and check if it can preserve both TRS and RS. (Note that terms proportional to $i \psi_{\sigma}^{a} \psi_{\sigma}^{b}$ for $\sigma=\uparrow$ or $\downarrow$ are irrelevant and only renormalize the Fermi velocities of edge Fermions.) Time-reversal symmetry requires $M_{a b}=M_{b a}$ while RS requires $M_{a b}=-M_{b a}$. Consequently, $M=0$ identically. In other words, it is impossible to gap the $N$ pairs of helical gapless Majorana edge states by considering the non-interacting fermion-bilinear terms for arbitrary $N$. This class of SCs is then characterized by an integer-valued $(\mathbb{Z})$ topological invariant (see below).

Microscopic models: Armed with insights from the edge theories, we now introduce an explicit mean-field SC Hamiltonian which respects both TRS and RS and which has non-trivial gapless helical Majorana edge states:

$$
\begin{aligned}
H_{\text {latt. }} & =\sum_{\langle i j\rangle \sigma}\left[-t c_{i \sigma}^{\dagger} c_{j \sigma}+H . c .\right]-\mu \sum_{i \sigma} c_{i \sigma}^{\dagger} c_{i \sigma} \\
& +\sum_{i}\left[\Delta\left(c_{i \uparrow}^{\dagger} c_{i+\hat{x} \uparrow}^{\dagger}+c_{i \downarrow}^{\dagger} c_{i+\hat{x} \downarrow}^{\dagger}\right)+H . c .\right] \\
& +\sum_{i}\left[i \Delta\left(c_{i \uparrow}^{\dagger} c_{i+\hat{y} \uparrow}^{\dagger}-c_{i \downarrow}^{\dagger} c_{i+\hat{y} \downarrow}^{\dagger}\right)+H . c .\right],
\end{aligned}
$$

where $c_{i \sigma}^{\dagger}$ are the fermion creation operators on site $i$ and $\sigma=\uparrow, \downarrow$ are spin indices. It is clear that the model above is invariant under RS $\mathcal{R}: c_{x, u, \sigma} \rightarrow\left(i \sigma^{x}\right)_{\sigma \sigma^{\prime}} c_{-x, y, \sigma^{\prime}}$ and TRS $\mathcal{T}: c_{i \sigma} \rightarrow\left(i \sigma^{y}\right)_{\sigma \sigma^{\prime}} c_{i \sigma^{\prime}}$ [28]. This model describes a $\mathrm{SC}$ with spin up $p_{x}+i p_{y}$ pairing and spin down $-p_{x}+i p_{y}$ pairing, and can describe a thin film of ${ }^{3} \mathrm{He}-\mathrm{B}$. On a cylinder with edges parallel to the $x$-direction, we (numerically) obtain the helical Majorana edge states, for $t=\Delta=1$ and for $-4<\mu<0$ or $0<\mu<+4$, say. These helical edge states are effectively described by the Hamiltonian (11). The $N$-flavor generalization of this model is straightforward.

Bulk topological invariant: There is a bulk topological invariant which guarantees, when non-interacting and when there is a translation symmetry, the stability of the edge modes of DIII+R topological SCs for arbitrary $N$. The construction is similar in spirit to the mirror Chern-number in 3D topological insulators protected by RS [29]. With the periodic boundary condition, the quadratic bulk Hamiltonian can be Fourier transformed as

$$
\begin{aligned}
& H=\sum_{0 \leq k_{x} \leq \pi} \sum_{k_{y}} \Psi_{k_{x}}^{\dagger}\left(k_{y}\right) \mathcal{H}_{k_{x}}\left(k_{y}\right) \Psi_{k_{x}}\left(k_{y}\right), \\
& \Psi_{k_{x}}^{\dagger}\left(k_{y}\right):=\left(c_{\uparrow, k}^{\dagger}, c_{\downarrow, k}^{\dagger}, c_{\uparrow,-k}, c_{\downarrow,-k}\right) .
\end{aligned}
$$

We then note at the reflection symmetric points $k_{x}=$ $\tilde{k}_{x}(=0$ and $\pi)$, the Bloch Hamiltonian $\mathcal{H}_{\tilde{k}_{x}}\left(k_{y}\right)$ commutes with $\mathcal{R}$, namely $\left[\mathcal{H}_{\tilde{k}_{x}}\left(k_{y}\right), J^{x}\right]=0$, where $J^{x}=$ $\operatorname{diag}\left(i \sigma^{x},-i \sigma^{x}\right)$. In other words, at $\tilde{k}_{x}$, the quadratic Hamiltonian conserves $S^{x}$. Combined with TRS, the quadratic Hamiltonian at $k_{x}=\tilde{k}_{x}$ can be written as

$$
H\left(\tilde{k}_{x}\right)=\sum_{k_{y}}\left(c_{+, k}^{\dagger}, c_{-,-k}\right) \overline{\mathcal{H}}_{\tilde{k}_{x}}\left(k_{y}\right)\left(\begin{array}{c}
c_{+, k} \\
c_{-,-k}^{\dagger}
\end{array}\right),
$$

where the subscripts \pm are eigenvalues of $\sigma^{x}$. Now, the Hamiltonian $\overline{\mathcal{H}}_{\tilde{k}}\left(k_{y}\right)$ above is in the AIII class with chiral symmetry $[15]$.

Following Ref. 15, gapped 1D quadratic Hamiltonians in symmetry class AIII are distinguished by an integer topological invariant, the winding number $\nu$. Thus, at each reflection symmetric momentum $k_{x}=\tilde{k}_{x}\left(\tilde{k}_{x}=0\right.$ and $\pi$ ), we can introduce an integer topological invariant ("the reflection winding number"), $\nu\left(\tilde{k}_{x}\right)$, the winding number of $\overline{\mathcal{H}}_{\tilde{k}_{x}}\left(k_{y}\right)$. We thus have two integral topological invariants, $\tilde{\nu}\left(k_{x}=0, \pi\right)$. The non-zero value of the invariant, $\tilde{\nu}\left(\tilde{k}_{x}\right) \neq 0$, guarantees the presence of $\left|\tilde{\nu}\left(k_{x}\right)\right|$ pairs of zero-energy Majorana states at $\tilde{k}_{x}$, when an edge is introduced along $x$-direction. In particular, when the invariant is non-zero $\tilde{\nu}\left(\tilde{k}_{x}\right) \neq 0$ at one of the reflection symmetric momenta $\left(\tilde{k}_{x}=0\right.$, say) and it is zero at the other $\left(\tilde{k}_{x}=\pi\right)$, this means there must be $\left|\tilde{\nu}\left(k_{x}\right)\right|$ branches of non-chiral edge modes. In general, the difference of the reflection winding number

$$
\tilde{\nu}\left(k_{x}=0\right)-\tilde{\nu}\left(k_{x}=\pi\right)
$$

tells us the number of non-chiral edge modes. 
Interaction effect: At the non-interacting level (and without disorder), the DIII+R SCs have $\mathbb{Z}$ topological classification as shown from both bulk and edge theories. Now, we consider the effect of symmetry-preserving interactions in the mean-field BdG Hamiltonian and check if the putative topological classification of $\mathbb{Z}$ is modified or not. Since gapless helical edge states are the hallmark of those topologically nontrivial SCs, we believe that it would be sufficient to check if the gapless helical edge states are stable against interactions while requiring there is no symmetry breaking induced by interactions [30].

For the case of topological invariant $N=8$ [more generally $N \equiv 0(\bmod 8)]$, we try to identify certain interactions that can destabilize the gapless edge states while preserving the symmetries of the bulk and the edge. For simplicity, we consider the helical edge modes of free fermions with the same Fermi velocities $v_{a}=v$

$$
H_{\text {free }}=v \int d x \sum_{a=1}^{8}\left[\psi_{\uparrow}^{a} i \partial_{x} \psi_{\uparrow}^{a}-\psi_{\downarrow}^{a} i \partial_{x} \psi_{\downarrow}^{a}\right],
$$

which is invariant under the global $\mathrm{SO}(8)$ rotations among the left-moving or right-moving Majorana fermions. Now, we consider interactions allowed by TRS and RS. One naturally starts with the following $\mathrm{SO}(8)$ symmetric interactions $H_{\mathrm{GN}}=H_{\text {free }}-$ $g \int d x\left(\sum_{a=1}^{8} i \psi_{\uparrow}^{a} \psi_{\downarrow}^{a}\right)^{2}$, where $g$ is the coupling constant. This theory is then the $\mathrm{SO}(8)$ Gross-Neveu (GN) model in $(1+1) \mathrm{D}$, which is exactly solvable [31]. Especially, the interaction is marginally relevant for $g>0$ : for arbitrary small interaction strength $g$ the ground state is gapped by spontaneously breaking the time reversal (or $\mathbb{Z}_{2}$ chiral) symmetry with the order parameter $\left\langle i \psi_{\uparrow}^{a} \psi_{\downarrow}^{a}\right\rangle \sim e^{-\pi /(v g)}$. There are twofold degenerate ground states at the edge. When $g<0$, the interaction is marginally irrelevant and the ground state remains gapless. In other words, the $\mathrm{SO}(8)$ symmetric GN interactions cannot result in a unique gapped ground state in the edge. We need to look for some other channel of interactions to fulfill this.

We follow the construction introduced in Ref. [19]. The non-interacting edge is described by the conformal field theory (CFT) of 8 free Majorana fermions, which is equivalent to the $\mathrm{SO}(8)_{1}$ Wess-Zumino-Witten model. The 8 fermion operators $\psi_{\sigma}^{a}(\sigma=\uparrow$ or $\downarrow)$ form the vector representation of $\mathrm{SO}(8)$. Moreover, the 16-dimensional spinor representation of $\mathrm{SO}(8)$ is reducible to two 8dimensional irreducible ones formed by spinor operators $\eta_{\sigma}^{a}$ and $\chi_{\sigma}^{a}, a=1, \ldots, 8$. The explicit forms of $\eta_{\sigma}^{a}$ and $\chi_{\sigma}^{a}$ are given by

$$
\exp \left[\frac{i}{2}\left( \pm \phi_{\sigma}^{1} \pm \phi_{\sigma}^{2} \pm \phi_{\sigma}^{3} \pm \phi_{\sigma}^{4}\right)\right]
$$

where $\phi_{\sigma}^{a}$ are boson fields obtained from bosonizing the system, $\psi_{\sigma}^{2 a-1} \pm i \psi_{\sigma}^{2 a}=e^{ \pm i \phi_{\sigma}^{a}}$. The number of minus sign in the exponent of Eq. (8) is even for $\eta^{a}$ but odd for $\chi^{a}$. Accidently, $\psi, \eta$ and $\chi$ all form 8-dimension representations of $\mathrm{SO}(8)$; they can actually be transformed into one another by the so-called triality symmetry of $\mathrm{SO}(8)$. It turns out that the spinor fields are useful to construct the interactions we desire.

To fully gap the edge states without spontaneously breaking any symmetry, we consider the following interactions

$$
\begin{aligned}
& H_{\mathrm{int}}=-\int d x {\left[A\left(\sum_{a=1}^{7} i \eta_{\uparrow}^{a} \eta_{\downarrow}^{a}\right)^{2}\right.} \\
&\left.+B\left(\sum_{a=1}^{7} i \eta_{\uparrow}^{a} \eta_{\downarrow}^{a}\right)\left(i \eta_{\uparrow}^{8} \eta_{\downarrow}^{8}\right)\right],
\end{aligned}
$$

which is $\mathrm{SO}(7)$-invariant and leaves $\eta_{\sigma}^{8}$ fixed. This $\mathrm{SO}(7)$ symmetric interaction is also local in terms of the original fermions $\psi_{\sigma}^{a}$. Indeed, a finite gap opens in the edge states while preserving the symmetries in question, as explicitly shown in Ref. [19] when $B<0$ and $2 A>B$. To understand this, let us first look at the limit $A \gg|B|$ which is the $\mathrm{SO}(7) \mathrm{GN}$ model plus free $\eta_{\sigma}^{8}$ fermions (when $B \rightarrow 0$ ). The chiral symmetry is broken by the $\mathrm{SO}(7)$ GN interactions with the order parameter $M=\left\langle i \sum_{a=1}^{7} i \eta_{\uparrow}^{a} \eta_{\downarrow}^{a}\right\rangle \neq 0$, which generates a mass term $i B M \eta_{\uparrow}^{8} \eta_{\downarrow}^{8}$ for $\eta^{8}$ fermions. Now, the $\eta^{8}$ fermions can be mapped to the transverse field Ising model with field strength $\left(h-h_{c}\right) \propto \pm B M$, where $h_{c}$ is the critical field strength in the transverse field Ising model which is equal to the Ising interaction strength. For $h>h_{c}$, its ground state is paramagnetic without any symmetry breaking; for $h<h_{c}$, the system spontaneously breaks the Ising symmetry resulting in twofold degenerate ground states. In other words, $B>0$ and $B<0$ lie in two different phases. Since $B=2 A>0$ is the $\mathrm{SO}(8)$ GN model which spontaneously break the chiral symmetry having twofold degenerate ground states, it is then clear that $B<0$ phase has a unique gapped ground state without breaking any symmetry.

It is worthwhile to understand more heuristically why $N=8$ is special. The edge theory with $N=8$ is qualitatively equivalent to the two-leg ladder electron model at half-filling. Since there are two electrons per unit cell for the two-leg ladder at half-filling, having a fully gapped ground state without breaking any symmetry is possible and expected [32]. To illustrate this, let us consider lattice Majorana fermions on $1 \mathrm{~d}$ chains described by the Hamiltonian [19], $H=u H_{1}+w H_{2}$ where $H_{1}$ is quadratic in lattice real fermion operators: $H_{1}=-(i / 2) \sum_{a=1}^{8} \sum_{j} s_{a} \lambda_{j}^{a} \lambda_{j+1}^{a}$, where $\left\{\lambda_{i}^{a}, \lambda_{j}^{b}\right\}=2 \delta_{i j}^{a b}$ and $s_{a}=\{1,1,-1,-1,1,1,-1,-1\}$. It is clear that $H_{1}$ is invariant under either TRS or RS, when we assume

$$
\begin{aligned}
& \mathcal{R}: \lambda_{j}^{a} \rightarrow(-1)^{a} \lambda_{-j}^{a+2}, \lambda_{j}^{a+2} \rightarrow-(-1)^{a} \lambda_{-j}^{a}, \\
& \mathcal{T}: \lambda_{j}^{a} \rightarrow \lambda_{j}^{a+2}, \lambda_{j}^{a+2} \rightarrow-\lambda_{j}^{a}, \quad a=1,2,5,6 .
\end{aligned}
$$

$H_{2}$ is an interaction, and given by $H_{2}=\sum_{j} W\left(\lambda_{j}^{a}\right)$ where $W\left(\lambda_{j}^{a}\right)$ is a four fermion interaction composed of eight 
Majorana fermions:

$$
\begin{aligned}
& W\left(\lambda^{a}\right)=+\lambda^{1} \lambda^{2} \lambda^{3} \lambda^{4}+\lambda^{1} \lambda^{2} \lambda^{5} \lambda^{6}+\lambda^{1} \lambda^{2} \lambda^{7} \lambda^{8} \\
& +\lambda^{3} \lambda^{4} \lambda^{5} \lambda^{6}+\lambda^{3} \lambda^{4} \lambda^{7} \lambda^{8}+\lambda^{5} \lambda^{6} \lambda^{7} \lambda^{8} \\
& -\lambda^{2} \lambda^{3} \lambda^{6} \lambda^{7}-\lambda^{1} \lambda^{4} \lambda^{5} \lambda^{8}+\lambda^{1} \lambda^{3} \lambda^{5} \lambda^{7}+\lambda^{2} \lambda^{4} \lambda^{6} \lambda^{8} \\
& -\lambda^{2} \lambda^{3} \lambda^{5} \lambda^{8}-\lambda^{1} \lambda^{4} \lambda^{6} \lambda^{7}-\lambda^{1} \lambda^{3} \lambda^{6} \lambda^{8}-\lambda^{2} \lambda^{4} \lambda^{5} \lambda^{7} .
\end{aligned}
$$

In the absence of the interaction term $w=0$, the lattice model is gapless, whose continuum limit is given by the Hamiltonian (7). When we switch on $w \neq 0$, the edge theory is gapped with unique ground state without breaking symmetries. This can be understood in the following way: the interaction $W$ can be written in terms of complex fermions

$$
\begin{aligned}
\left(\lambda^{1}+i \lambda^{2}\right) / 2 & =c_{1 \uparrow}, \quad\left(\lambda^{3}-i \lambda^{4}\right) / 2=c_{1 \downarrow}, \\
\left(-\lambda^{5}+i \lambda^{6}\right) / 2 & =c_{2 \uparrow}, \quad\left(\lambda^{7}+i \lambda^{8}\right) / 2=c_{2 \downarrow},
\end{aligned}
$$

as follows:

$$
W\left(\lambda_{j}^{a}\right)=16 \boldsymbol{S}_{j, 1} \cdot \boldsymbol{S}_{j, 2}+2\left(n_{j, 1}-1\right)^{2}+2\left(n_{j, 2}-1\right)^{2}-2,
$$

where $\boldsymbol{S}_{1,2}$ and $n_{1,2}$ is the spin and the fermion number operator for $c_{1,2 s}(s=\uparrow / \downarrow)$. With this interaction, the charge degrees of freedom will be frozen by Mott physics. The exchange interaction $\boldsymbol{S}_{1} \cdot \boldsymbol{S}_{2}$ (the "rung-exchange" interaction) realizes the rung-single phase which has a unique ground state without breaking any symmetry.

Discussion: We have discussed interaction effects on topological SCs in 2D protected by TRS and RS. This is a non-trivial example in 2D where topological classification of ground states are dramatically altered by the interaction effect. In a separate paper, we plan on a more systematic study on topological insulators and SCs protected by RS in addition to other possible discrete symmetries 33,34 ].

We close with discussion on disorder effects: In the ten-fold classification of topological insulators and SCs, it has been proved useful to consider the boundary (edge, surface, etc.) Anderson localization problem: For a topological bulk, one should find a boundary mode which is completely immune to disorder. In turn, once one finds such "Anderson delocalization" at the boundary, it means there is a topologically non-trivial bulk. Not only this bulk-boundary correspondence can be used to find and classify bulk topological phases in the absence of disorder, it immediately tells us such topological phases are stable against disorder. For topological phases protected by a set of spatial symmetries, stability against disorder is, in general, not trivial, since spatial inhomogeneity does not respect the spatial symmetries. One can still consider, however, situations where the spatial symmetries are preserved on average. The effects of disorder in $N$-channel quantum wires in symmetry class DIII, which are from our point of view the edge theory of the DIII $+\mathrm{R}$ topological SC with the topological integer $(=N)$, have been studies [35]. It is known that there is an even-odd effect in $N$ : the mean conductance decreases algebraically as $L^{-1 / 2}$ with the length of the wire $L$ for odd $N$, whereas it decays exponentially with $L$ for even $N$. Correspondingly, for symmetry class DIII with $N$ odd the density of states shows the Dyson singularity. This implies that disorder simply reduces the $\mathbb{Z}$ topological classification of $\mathrm{DIII}+\mathrm{R}$ SCs to the $\mathbb{Z}_{2}$ classification, which is the same as the topological classification of 2D DIII SCs in the periodic table. In a separate paper [33] we will report the other cases where the $\mathbb{Z}$ topological classification is reduces to the $\mathbb{Z}_{2}$ classification, which is not related to the existing topological class in the periodic table.

Note added: Recently, some other papers, a couple of works that deal with similar topic have appeared [36 38].

Acknowledgements: We would like to thank EunAh Kim, Steve Kivelson, Dung-Hai Lee, and Shou-Cheng Zhang for helpful discussion, and in particular XiaoLiang Qi for sharing his results with us prior to its arXiv submission. This work is supported in part by Tsinghua Startup Fund and by US NSF Grant DMR-0904264.

[1] X.-G. Wen, Phys. Rev. B 40, 7387 (1989).

[2] The Quantum Hall Effect, edited by R. E. Prange and S. M. Girvin (Springer, New York, 1987).

[3] M. Z. Hasan and C. L. Kane, Rev. Mod. Phys. 82, 3045 (2010).

[4] X.-L. Qi and S.-C. Zhang, Rev. Mod. Phys. 83, 1057 (2011).

[5] C. L. Kane and E. J. Mele, Phys. Rev. Lett. 95, 146802 (2005); C. L. Kane and E. J. Mele, Phys. Rev. Lett. 95, 226801 (2005).

[6] B. A. Bernevig and S.-C. Zhang, Phys. Rev. Lett. 96, 106802 (2006).

[7] R. Roy, Phys. Rev. B 79, 195321 (2009).

[8] J. E. Moore and L. Balents, Phys. Rev. B 75, 121306(R) (2007).

[9] L. Fu, C. L. Kane, and E. J. Mele, Phys. Rev. Lett. 98, 106803 (2007).

[10] R. Roy, Phys. Rev. B 79, 195322 (2009).

[11] L. Fu and C. L. Kane, Phys. Rev. B 76, 045302 (2007).

[12] X.-L. Qi and T. Hughes and S.-C. Zhang, Phys. Rev. B 78, 195424 (2008).

[13] See, Y. Wada et al., Phys. Rev. B 78, 214516 (2008), S. Murakawa et al., Phys. Rev. Lett. 103, 155301 (2009), S. Murakawa et al., J. Phys. Soc. Jpn. 80, 013602 (2011), and references therein.

[14] Here, a "non-interacting" SC means it is described by its mean-field Hamiltonian even though the SC comes from some sort of interactions.

[15] A. P. Schnyder, S. Ryu, A. Furusaki, and A. W. W. Ludwig, Phys. Rev. B 78, 195125 (2008).

[16] S. Ryu, A. Schnyder, A. Furusaki and A. W. W. Ludwig, New J. Phys. 12, 065010 (2010).

[17] A. Kitaev, AIP Conf. Proc. 1134, 22 (2009).

[18] C. Wu, B. A. Bernevig, and S.-C. Zhang, Phys. Rev. Lett. 
96, 106401 (2006); C. Xu and J. E. Moore, Phys. Rev. B 73, 045322 (2006).

[19] L. Fidkowski and A. Kitaev, Phys. Rev. B 81, 134509 (2010).

[20] L. Fidkowski and A. Kitaev, Phys. Rev. B 83, 075103 (2011).

[21] A. M. Turner, F. Pollmann, and E. Berg, Phys. Rev. B 83, 075102 (2011).

[22] S. Ryu, J. E. Moore, and A. W. W. Ludwig, Phys. Rev. B 85, 045104 (2012).

[23] Z. Wang, X.-L. Qi, and S.-C. Zhang, Phys. Rev. B 84, 014527 (2011).

[24] X. Chen, Z.-C. Gu, Z.-X. Liu, and X.-G. Wen, Phys. Rev. B 87, 155114 (2013)..

[25] Z.-C. Gu and X.-G. Wen, arXiv:1201.2648

[26] X.-L. Qi, New J. Phys. 15, 065002 (2013).

[27] S. Ryu and S.-C. Zhang, Phys. Rev. B 85, 245132 (2012).

[28] Note that the reflection $\mathcal{R}$ is given by $i \sigma^{x}$ in the bulk. When projected on the edge, $\mathcal{R}$ is given by $i \sigma^{y}$, as required by the reality nature of Majorana fermions on the edge and the condition $\mathcal{R}^{2}=-1$.

[29] J. C. Y. Teo, L. Fu, and C. L. Kane, Phys. Rev. B 78, 045426 (2008).
[30] To explicitly show that the topological classification in the interacting DIII $+\mathrm{R}$ SCs is $\mathbb{Z}_{8}$, constructing an adiabatic path connecting the $N=8$ model to the $N=0$ model without closing the bulk gap should be needed, as what Fidkowski and Kitaev did in [19] for the $(1+1) \mathrm{D}$ BDI SCs, even though we believe that the classification argued from the edge consideration is sufficient.

[31] R. Shankar, Phys. Rev. Lett. 46, 379 (1981).

[32] H.-H. Lin, L. Balents, and M. P. A. Fisher, Phys. Rev. B 58, 1794 (1998).

[33] C.-K. Chiu, H. Yao, and S. Ryu, arXiv:1303.1843.

[34] A systematic study of $1 \mathrm{D}$ edge theories of a 2D bulk was also given recently in D. Bernard, E. -A. Kim, and A. LeClair, Phys. Rev. B 86, 205116 (2012).

[35] See, for example, P. Brouwer, A. Furusaki, C. Mudry, and S. Ryu, BUTSURI 60, 935 (2005) (in Japanese) (English version: arXiv:cond-mat/0511622).

[36] Y. Ueno, A. Yamakage, Y. Tanaka, and M. Sato, arXiv:1303.0202

[37] F. Zhang, C. L. Kane, and E. J. Mele, arXiv:1303.4144.

[38] T. Morimoto and A. Furusaki, arXiv:1306.2505 